\title{
Multiple logarithms, algebraic cycles and trees
}

\author{
H. Gangl ${ }^{1}$, A.B. Goncharov ${ }^{2}$, and A. Levin ${ }^{3}$ \\ 1 MPI für Mathematik, Vivatsgasse 7, D-53111 Bonn, Germany \\ 2 Brown University, Box 1917, Providence, RI 02912, USA \\ 3 Institute of Oceanology, Moscow, Russia
}

This is a short exposition - mostly by way of the toy models "double logarithm" and "triple logarithm" - which should serve as an introduction to the article [3] in which we establish a connection between multiple polylogarithms, rooted trees and algebraic cycles.

\section{Introduction}

The multiple polylogarithm functions were defined in [5] by the power series

$$
L i_{n_{1}, \ldots, n_{m}}\left(z_{1}, \ldots, z_{m}\right)=\sum_{0<k_{1}<\cdots<k_{m}} \frac{z_{1}^{k_{1}}}{k_{1}^{n_{1}}} \frac{z_{2}^{k_{2}}}{k_{2}^{n_{2}}} \ldots \frac{z_{m}^{k_{m}}}{k_{m}^{n_{m}}} \quad\left(z_{i} \in \mathbb{C},\left|z_{i}\right|<1\right) .
$$

They admit an analytic continuation to a Zariski open subset of $\mathbb{C}^{m}$. Putting $m=1$ in this definition, we recover the classical polylogarithm function. Putting $n_{1}=\ldots=n_{m}=1$, we get the multiple logarithm function.

Let $x_{i}$ be complex numbers. Recall that an iterated integral is defined as

$$
I\left(x_{0} ; x_{1}, \ldots, x_{m} ; x_{m+1}\right)=\int_{\Delta_{\gamma}} \frac{d t_{1}}{t_{1}-x_{1}} \wedge \cdots \wedge \frac{d t_{m}}{t_{m}-x_{m}},
$$

where $\gamma$ is a path from $x_{0}$ to $x_{m+1}$ in $\mathbb{C}-\left\{x_{1}, \ldots, x_{m}\right\}$, and the cycle of integration $\Delta_{\gamma}$ consists of all $m$-tuples of points $\left(\gamma\left(t_{1}\right), \ldots, \gamma\left(t_{m}\right)\right)$ with $t_{i} \leqslant t_{j}$ for $i<j$.

Multiple polylogarithms can be written as iterated integrals (cf. loc.cit.). In particular, the iterated integral representation of the multiple logarithm function is given as

$$
L i_{1, \ldots, 1}\left(z_{1}, \ldots, z_{m}\right)=(-1)^{m} I\left(0 ; x_{1}, \ldots, x_{m} ; 1\right),
$$

where we set 


$$
x_{1}:=\left(z_{1} \cdots z_{m}\right)^{-1}, \quad x_{2}:=\left(z_{2} \cdots z_{m}\right)^{-1}, \quad \ldots \quad, x_{m}:=z_{m}^{-1} .
$$

Observe that in (3) the parameters $x_{1}, \ldots, x_{m}$ are non-zero. Many properties of the iterated integrals will change if we put some of the $x_{i}$ 's equal to zero, which is why the study of multiple polylogarithms cannot be directly reduced to investigating multiple logarithms only.

Notation: We will use the notation $I_{1, \ldots, 1}\left(x_{1}, \ldots, x_{m}\right)$ for $I\left(0 ; x_{1}, \ldots, x_{m} ; 1\right)$ in order to emphasize that the $x_{i}$ are non-zero.

In the paper [1], Bloch and Kříž defined an algebraic cycle realization of the classical polylogarithm function. The goal of our project [3] was to develop a similar construction for multiple polylogarithms. In this paper we explain how to do this in the case of multiple logarithms, with special emphasis on the toy examples of the double and triple logarithm.

The structure of the paper is as follows. Let $F$ be a field. In Section 2 we recall Bloch's differential graded algebra $\mathcal{Z}^{\bullet}(F, \bullet)$ of cubical algebraic cycles. In Section 3 we define, for a set $R$, another differential graded algebra $\mathcal{T}_{\bullet}^{\bullet}(R)$, built from $R$-decorated rooted forests. A very similar differential graded algebra, for non-rooted forests, was introduced in [7].

In Section 4 we relate these two DGA's in the case when $R=F^{\times}$. More precisely, we define a subalgebra $\widetilde{\mathcal{T}}_{\bullet}^{\bullet}\left(F^{\times}\right)$of $\mathcal{T}_{\bullet}^{\bullet}\left(F^{\times}\right)$by imposing some explicit genericity condition on the decoration. Then we construct a map of graded Hopf algebras

$$
\varphi: \widetilde{\mathcal{T}}_{\bullet}^{\bullet}\left(F^{\times}\right) \longrightarrow \mathcal{Z}^{\bullet}(F, \bullet) .
$$

In Section 5 we introduce the second important ingredient of our construction: given a collection of elements $x_{1}, \ldots, x_{m} \in F^{\times}$, we define an element

$$
\tau\left(x_{1}, \ldots, x_{m}\right) \in \mathcal{T}_{\bullet}^{\bullet}\left(F^{\times}\right)
$$

Under certain explicit conditions on the $x_{i}$ 's, it belongs to $\tilde{\mathcal{T}}_{\bullet}^{\bullet}\left(F^{\times}\right)$. Then the algebraic cycle $\varphi \tau\left(x_{1}, \ldots, x_{m}\right)$ corresponds to the multiple logarithm (2). In Section 6, using ideas from [1] concerning the Hodge realization for $\mathcal{Z}^{\bullet}(\mathbb{C}, \bullet)$, we show by way of example how to get the original multivalued analytic functions (2) from the constructed cycles.

Acknowledgement. This work has been done while we enjoyed the hospitality of the MPI (Bonn). We are grateful to the MPI for providing ideal working conditions and support. A.G. was supported by the NSF grant DMS0099390. A.L. was partially supported by the grant RFFI 04-01-00642.

\section{Cubical algebraic cycles}

Let $F$ be a field. Following [2], we define the algebraic 1-cube $\square_{F}$ as a pair

$$
\square_{F}=\left(\mathbb{P}_{F}^{1} \backslash\{1\} \simeq \mathbb{A}_{F}^{1},(0)-(\infty)\right)
$$


Here we consider the standard coordinate $z$ on the projective line $\mathbb{P}_{F}^{1}$ and remove from it the point 1 . Furthermore, $(0)-(\infty)$ denotes the divisor defined by the two points 0 and $\infty$. The algebraic $n$-cube is defined by setting $\square_{F}^{n}=$ $\left(\square_{F}\right)^{n}$.

Bloch defined the cycle groups

$$
\begin{gathered}
\mathcal{C}^{p}(F, n)=\mathbb{Z}[\text { admissible closed irreducible subvarieties over } F, \\
\text { of codimension } \left.\left.p \text { in } \square_{F}^{n}\right\}\right] .
\end{gathered}
$$

Here a cycle is called admissible if it intersects all the faces (of any codimension) of $\square_{F}^{n}$ properly, i.e., in codimension $p$ or not at all. Consider the semidirect product of the symmetric group $S_{n}$ and the group $(\mathbb{Z} / 2 \mathbb{Z})^{n}$, acting by permuting and inverting the coordinates in $\square_{F}^{n}$. Let $\varepsilon_{n}$ be the sign representation of this group. The group $\mathcal{Z}^{p}(F, n)$ is defined as the coinvariants of this group acting on $\mathcal{C}^{p}(F, n) \otimes \varepsilon_{n}$. Bloch showed that these groups, for a fixed $p$, form a complex

$$
\cdots \rightarrow \mathcal{Z}^{p}(F, n) \stackrel{\partial}{\rightarrow} \mathcal{Z}^{p}(F, n-1) \rightarrow \ldots
$$

where the differential $\partial$ is given by

$$
\partial=\sum_{i=1}^{n}(-1)^{i-1}\left(\partial_{0}^{i}-\partial_{\infty}^{i}\right)
$$

and $\partial_{\varepsilon}^{i}$ denotes the operator which is given by the intersection with the coordinate hyperplane $\left\{z_{i}=\varepsilon\right\}, \varepsilon \in\{0, \infty\}$.

The concatenation of coordinates, followed by the corresponding projection under the alternation of the coordinates, provides a product on algebraic cycles, and together with the above one gets

Proposition 2.1 (Bloch) The algebraic cycle groups $\mathcal{Z}^{p}(F, n)$ associated to a given field $F$ provide a differential graded algebra $\mathcal{Z}^{\bullet}(F, \bullet)=\sum_{p, n} \mathcal{Z}^{p}(F, n)$.

Example 2.2 For any element a in $F$, one can associate such a cubical algebraic cycle corresponding to the dilogarithm $\mathrm{Li}_{2}(a)$. This cycle has been given by Totaro as the image of the map

$$
\begin{aligned}
\varphi_{a}: \mathbb{P}_{F}^{1} & \rightarrow\left(\mathbb{P}_{F}^{1}\right)^{3}, \\
t & \mapsto\left(t, 1-t, 1-\frac{a}{t}\right),
\end{aligned}
$$

restricted to the algebraic cube $\square_{F}^{3}$ : we write

$$
C_{a}:=\left[t, 1-t, 1-\frac{a}{t}\right]:=\varphi_{a}\left(\mathbb{P}_{F}^{1}\right) \cap \square_{F}^{3} .
$$

The cycle $C_{a}$ belongs to the group $\mathcal{Z}^{2}(F, 3)$. One has 


$$
\partial C_{a}=[a, 1-a] \in \square_{F}^{2}
$$

(only $\partial_{0}^{3}$ gives a non-empty contribution). The same computation shows that $C_{a}$ is in fact admissible. Observe the apparent similarity with the formula $d L i_{2}(a)=-\log (1-a) d \log (a)$ for the differential of the dilogarithm.

Example 2.3 Recall (cf. [6]) that the double logarithm is defined via the power series

$$
L i_{1,1}(x, y)=\sum_{0<m<n} \frac{x^{m}}{m} \frac{y^{n}}{n} \quad(|x|,|y|<1, x, y \in \mathbb{C}) .
$$

Its differential is computed as follows

$$
\begin{aligned}
d L i_{1,1}(x, y) & =\sum_{0<m<n} x^{m-1} \frac{y^{n}}{n} d x+\sum_{0<m<n} \frac{x^{m}}{m} y^{n-1} d y \\
& =\sum_{n>0} \frac{1-x^{n-1}}{1-x} \frac{y^{n}}{n} d x+\sum_{0<m} \frac{x^{m}}{m} \frac{y^{m} d y}{1-y} \\
& =L i_{1}(y) \frac{d x}{1-x}-L i_{1}(x y) \frac{d x}{x(1-x)}+L i_{1}(x y) \frac{d y}{1-y} \\
& =L i_{1}(y) d L i_{1}(x)-L i_{1}(x y) d L i_{1}\left(\frac{1}{x}\right)+L i_{1}(x y) d L i_{1}(y) .
\end{aligned}
$$

The cycle

$$
C_{a, b}:=\left[1-t, 1-\frac{a b}{t}, 1-\frac{b}{t}\right] \in \mathcal{Z}^{2}(F, 3)
$$

will play the role of the double logarithm $L i_{1,1}(a, b)$ among the algebraic cycles. Its boundary is readily evaluated as

$$
\partial C_{a, b}=\underbrace{[1-a b, 1-b]}_{\text {from } z_{1}=0}-\underbrace{\left[1-a b, 1-\frac{1}{a}\right]}_{\text {from } z_{2}=0}+\underbrace{[1-b, 1-a]}_{\text {from } z_{3}=0} \in \mathcal{Z}^{2}(F, 2)
$$

whose individual terms are already very reminiscent of the three terms in (4).

Observe that, setting $x_{1}=(a b)^{-1}, x_{2}=b^{-1}$, we can rewrite $C_{a, b}$ as

$$
Z_{x_{1}, x_{2}}:=\left[1-\frac{1}{u}, 1-\frac{u}{x_{1}}, 1-\frac{u}{x_{2}}\right]
$$

whose constants $x_{1}, x_{2}$ are chosen to match the iterated integral form $I_{1,1}\left(x_{1}, x_{2}\right)$ of $L i_{1,1}(a, b)$. In the following, we will deal with cycles in the $Z_{\ldots}$. -form. (Note that the change of variable $t=u^{-1}$ does not change the cycle.)

Below we will explain how to generalize this definition for the case of multiple logarithms. 


\section{The differential graded algebra of $\boldsymbol{R}$-deco forests}

In this paper a plane tree is a finite tree whose internal vertices are of valency $\geqslant 3$, and where at each vertex a cyclic ordering of the incident edges is given. We assume that all the other vertices are of valency 1 , and call them external vertices. A plane tree is planted if it has a distinguished external vertex of valency 1 , called its root. A forest is a disjoint union of trees.

\subsection{The orientation torsor}

Recall that a torsor under a group $G$ is a set on which $G$ acts freely transitively.

Let $S$ be a finite (non-empty) set. We impose on the set of orderings of $S$ an equivalence relation, given by even permutations of the elements. The equivalence classes form a 2 -element set $\mathrm{Or}_{S}$. It has an obvious $\mathbb{Z} / 2 \mathbb{Z}$-torsor structure and is called the orientation torsor of $S$.

Definition 3.1 Let $F$ be a plane forest. The orientation torsor of $F$ is the orientation torsor of the set of its edges.

Observe that once we have chosen an edge ordering in a plane tree $T$, e.g., by fixing a root, there is a canonical orientation on $T$.

\subsection{The algebra of $R$-deco forests}

Definition 3.2 Let $R$ be a set. An $R$-deco tree is a planted plane tree with a map, called $R$-decoration, from its external vertices to $R$. An $R$-deco forest is a disjoint union of $R$-deco trees.

Remark 3.3 1. There is an obvious induced direction for each edge in an $R$-deco tree, away from the root.

2. There is an ordering of the edges, starting from the root edge, which is induced by the cyclic ordering of edges at internal vertices.

Our convention for drawing the trees is that the cyclic ordering of edges around internal vertices is displayed in counterclockwise direction.

Example 3.4 We draw an $R$-deco tree $T$ with root vertex decorated by $x_{4} \in$ $R$; its other external vertices are decorated by $x_{1}, x_{2}, x_{3} \in R$. The abovementioned ordering of the edges $e_{i}$ coincides with the natural ordering of their indices, while the direction of the edges (away from the root) is indicated by small arrows along the edges. 


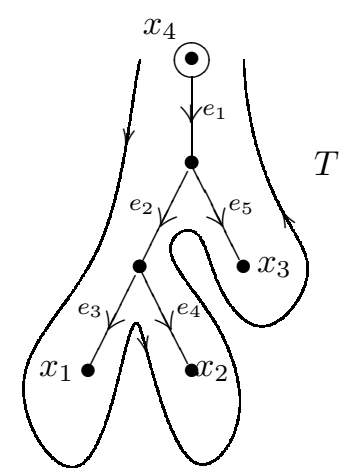

Figure 1: An $R$-deco tree $T$ with root vertex decorated by $x_{4}$.

Definition 3.5 For a set $R$, the $\mathbb{Q}$-vector space $\mathcal{T}_{\bullet}(R)$ is generated by the elements $(F, \omega)$, where $F$ is an $R$-deco forest and $\omega$ an orientation on it, subject to the relation $(F,-\omega)=-(F, \omega)$.

We define the grading of an $R$-deco tree $T$ by

$$
e(T)=\#\{\text { edges of } T\}
$$

and extend it to forests by linearity: $e\left(F_{1} \sqcup F_{2}\right):=e\left(F_{1}\right)+e\left(F_{2}\right)$. It provides the vector space $\mathcal{T}_{\bullet}(R)$ with a natural grading.

We define the algebra structure $\star$ on $\mathcal{T}_{\bullet}(R)$ by setting

$$
\left(F_{1}, \omega_{1}\right) \star\left(F_{2}, \omega_{2}\right):=\left(F_{1} \sqcup F_{2}, \omega_{1} \otimes \omega_{2}\right)
$$

It makes $\mathcal{T}_{\bullet}(R)$ into a graded commutative algebra, called the $R$-deco forest algebra $\mathcal{T}_{\bullet}(R)$.

Let $V_{\bullet}(R)$ be the graded $\mathbb{Q}$-vector space with basis given by $R$-deco trees, with the above grading.

Lemma 3.6 The algebra $\mathcal{T}_{\bullet}(R)$ is the free graded commutative algebra generated by the graded vector space $V_{\bullet}(R)$.

So the basis elements of $V_{\bullet}(R)$ commute in the $R$-deco forest algebra via the rule

$$
\left(T_{1}, \omega_{1}\right) \star\left(T_{2}, \omega_{2}\right)=(-1)^{e\left(T_{1}\right) e\left(T_{2}\right)}\left(T_{2}, \omega_{2}\right) \star\left(T_{1}, \omega_{1}\right) .
$$

\subsection{The differential}

A differential on $\mathcal{T}_{\bullet}(R)$ is a map

$$
d: \mathcal{T}_{\bullet}(R) \longrightarrow \mathcal{T}_{\bullet-1}(R)
$$

satisfying $d^{2}=0$ and the Leibniz rule. Since, by Lemma 3.6, $\mathcal{T}_{\bullet}(R)$ is a free graded commutative algebra, it is sufficient to define it on the algebra 
generators, that is on the elements $(T, \omega)$, where $T$ is an $R$-deco tree and $\omega$ is an orientation of $T$.

The terms in the differential of a tree $T$ arise by contracting an edge of $T$ they fall into two types, according to whether the edge is internal or external. We will need the notion of a splitting.

Definition 3.7 $A$ splitting of a tree $T$ at an internal vertex $v$ is the disjoint union of the trees which arise as $T_{i} \cup v$ where the $T_{i}$ are the connected components of $T \backslash v$.

Further structures on $T$, e.g. a decoration at $v$, planarity of $T$ or an ordering of its edges, are inherited for each $T_{i} \cup v$. Also, if $T$ has a root $r, v$ plays the role of the root for all $T_{i} \cup v$ which do not contain $r$.

Definition 3.8 Let e be an edge of a tree $T$. The contraction of $T$ along $e$, denoted $T / e$, is given as follows:

1. If $e$ is an internal edge, then $T / e$ is again a tree: it is the same tree as $T$ except that $e$ is contracted and the incident vertices $v$ and $v^{\prime}$ of $e$ are identified to a single vertex.

2. If $e$ is an external edge, then $T / e$ is obtained as follows: first we contract the edge $e$ to a vertex $w$ and then we perform a splitting at $w$.

Two typical examples are given below: in Figure 2 we contract a leaf, i.e. an external vertex which is not the root vertex, and in Figure 3 the root vertex is contracted.
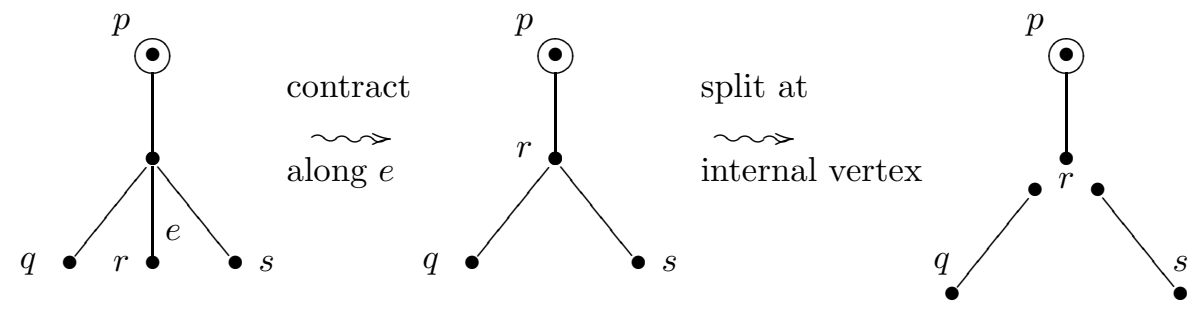

Figure 2: Contracting a leaf.
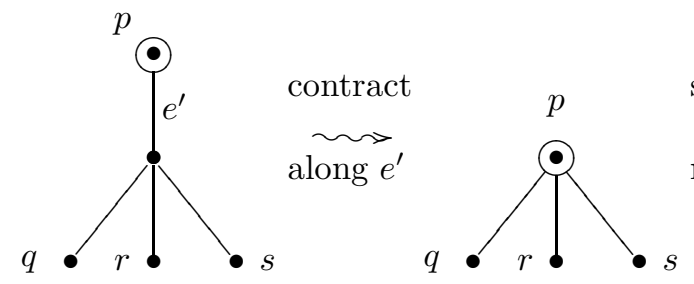

split at
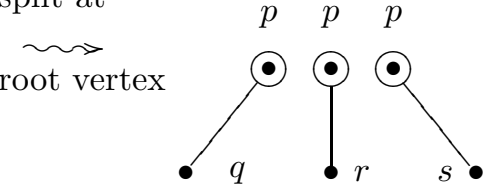

Figure 3: Contracting the root edge. 
Let $S$ be a finite set, and $\mathrm{Or}_{S}$ the orientation torsor of $S$. We present its elements as $s_{1} \wedge \ldots \wedge s_{n}$, where $n=|S|$. Now given an element $s \in S$ and $\omega \in \mathrm{Or}_{S}$, we define an element $i_{s} \omega \in \mathrm{Or}_{S-s}$ as follows:

$$
i_{s} \omega:=s_{2} \wedge \ldots \wedge s_{n} \quad \text { if } \omega=s \wedge s_{2} \wedge \ldots \wedge s_{n} .
$$

Definition 3.9 Let $T$ be a finite tree with set of edges $E$, and let $\omega$ be an orientation of $T$. The differential on $(T, \omega)$ is defined as

$$
d:(T, \omega) \mapsto \sum_{e \in E}\left(T / e, i_{e} \omega\right) .
$$

Example 3.10 The simplest non-trivial example for the differential of an $F^{\times}$-deco tree, where $F^{\times}$is the multiplicative group of a field $F$, can be seen on a tree with one internal vertex, as given in Figure 4. Here we choose the $F^{\times}$-decoration $\left(x_{1}, x_{2}\right)$ with $x_{1}, x_{2} \in F^{\times}$for the leaves and the decoration 1 for the root:

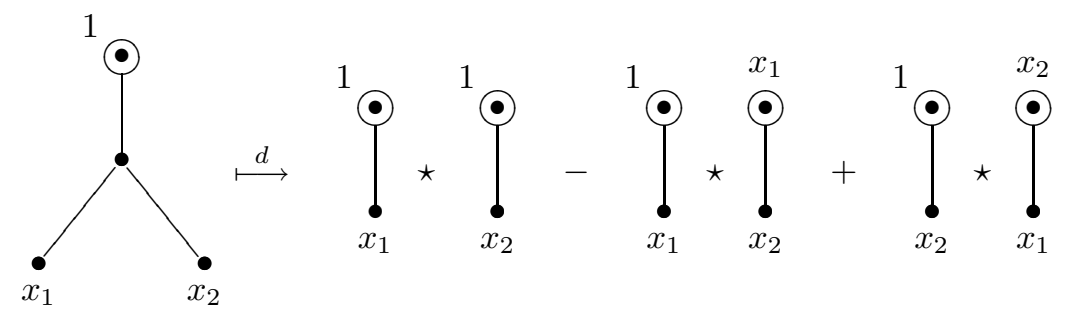

Figure 4: The differential on a tree with one internal vertex.

For the drawings, we use the canonical ordering of edges for $F^{\times}$-deco trees and the induced ordering for forests which arise from a splitting. The ordering of the forest encodes its orientation, i.e. the choice of an element in the orientation torsor.

Remark 3.11 There is a $\mathbb{Z}$-bigrading on $\mathcal{T}_{\bullet}(R)$ given by the groups

$$
\mathcal{T}_{n}^{p}(R)=\mathbb{Z}[\{R \text {-deco forests with } n \text { edges and } p \text { leaves }\}] .
$$

It will correspond below to the bigrading on the cycle groups $\mathcal{Z}^{p}(F, n)$.

For a set $R$, put

$$
\mathcal{T}_{\bullet}^{\bullet}(R):=\bigoplus_{p \geqslant 0} \bigoplus_{0 \leqslant n \leqslant p} \mathcal{T}_{n}^{p}(R)
$$

With the above definitions, we have:

Proposition $3.12\left(\mathcal{T}_{\bullet}^{\bullet}(R), d\right)$ is a bigraded, differential graded algebra. 


\section{Mapping forests to algebraic cycles}

In the special case where $R=F^{\times}$, the multiplicative group of a field $F$, we can establish the connection between the two differential graded algebras above, and Theorem 4.2 below gives the main result of this paper.

It turns out that the admissibility condition on algebraic cycles mentioned above forces us to restrict to a subalgebra of $\mathcal{T}^{\bullet}$, which we now describe.

Definition 4.1 We call an $R$-deco tree generic if all the individual decorations of external vertices are different.

We denote the subalgebra of $\mathcal{T}_{\bullet}^{\bullet}(R)$ generated by generic $R$-deco trees by $\tilde{\mathcal{T}}_{\bullet}^{\bullet}(R)$.

One of our key results is the following statement:

Theorem 4.2 For a field $F$, there is a natural map of differential graded algebras

$$
\tilde{\mathcal{T}}_{\bullet}^{\bullet}\left(F^{\times}\right) \rightarrow \mathcal{Z}^{\bullet}(F, \bullet) .
$$

It is given by the map in Definition 4.3 below.

Definition 4.3 The forest cycling map for a field $F$ is the map $\varphi$ from $\mathcal{T}_{\bullet}\left(F^{\times}\right)$to (not necessarily admissible) cubical algebraic cycles over $F$ given on generators, i.e. $F^{\times}$-deco trees, as follows:

1. to each internal vertex $v$ of $T$ we associate a decoration consisting of an independent ("parametrizing") variable;

2. to each edge with (internal or external) vertices $v$ and $w$ equipped with respective decorations $y_{v}$ and $y_{w}$ (variables or constants) we associate the expression $\left[1-y_{w} / y_{v}\right]$ as a parametrized coordinate in $\mathbb{P}_{F}^{1}$;

3. choosing an ordering of edges of $T$ corresponding to $\omega$, we concatenate all the respective coordinates produced in the previous step.

This somewhat lengthy description is easily understood by looking at an example. We will denote the concatenation product for algebraic cycles by $*$,

and we encode the expression $1-\frac{x}{y}$, for $x, y$ in a field, by the following picture

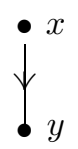

Example 4.4 Let us consider the forest cycling map $\varphi$ for the following $R$ deco tree $(T, \omega)$, where the orientation $\omega$ is given by $e_{1} \wedge e_{2} \wedge e_{3}$ (we leave out the arrows since the edges are understood to be directed away from the root): 


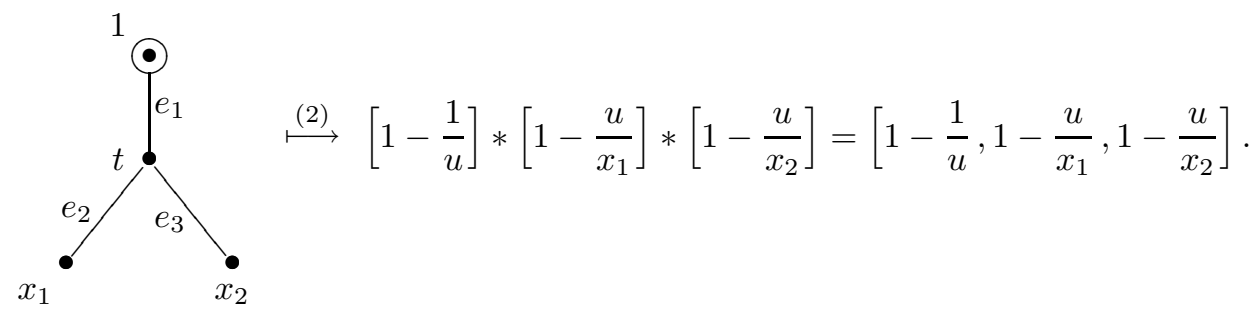

Figure 5: The forest cycling map on a tree with one internal vertex.

This cycle, as already mentioned, corresponds to the double logarithm $I_{1,1}\left(x_{1}, x_{2}\right)$, as we will see in Section 6.

Lemma 4.5 Each generic $F^{\times}$-deco tree maps to an admissible cycle in $\mathcal{Z}^{\bullet}(F, \bullet)$.

For a proof, we refer to [3]; the main idea is that at each internal vertex we have at least one incoming and one outgoing edge, which implies that their respective coordinates in the associated cycle cover up for each other.

\section{The algebraic cycle corresponding to the multiple logarithm}

Definition 5.1 Let $\left\{x_{1}, \ldots, x_{m}\right\}$ be a collection of distinct elements of $F^{\times} \backslash$ $\{1\}$. Then $\tau\left(x_{1}, \ldots, x_{m}\right)$ is the sum of all trivalent $F^{\times}$-deco trees with $m$ leaves whose $F^{\times}$-decoration is given by $\left(x_{1}, x_{2}, \ldots, x_{m-1}, x_{m}\right)$, while the root is decorated by 1.

Recall that the number of such trees is given by the Catalan number $\frac{1}{m}\left(\begin{array}{c}2(m-1) \\ m-1\end{array}\right)$.

Combining Definition 5.1 with the forest cycling map $\varphi$, we get the cycles corresponding to the multiple logarithms. For $m=2$, the tree $\tau\left(x_{1}, x_{2}\right)$ is given by the tree on the left of Figure 5 , where $x_{3}$ is put equal to 1 .

Example 5.2 The simplest example where the sum of trees consists of more than a single term appears when $m=3$ : 


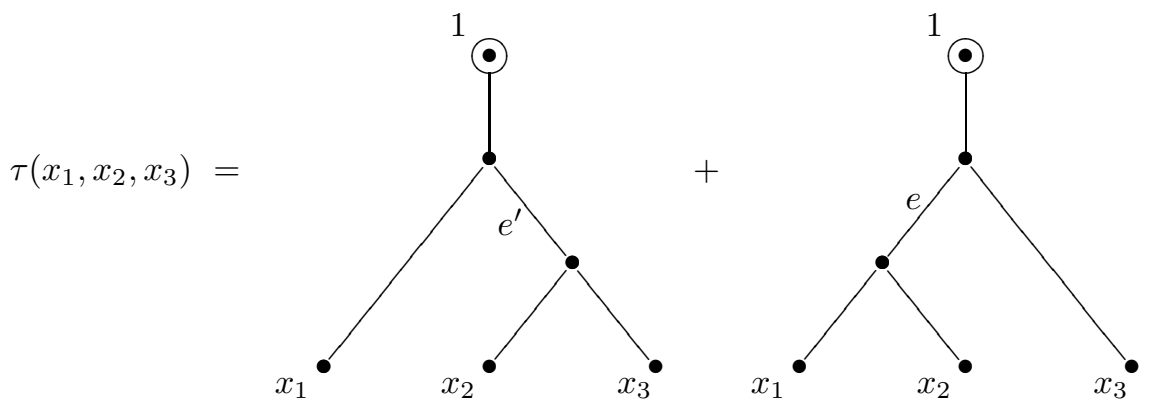

Figure 6: The sum of trees corresponding to the triple logarithm $I_{1,1,1}\left(x_{1}, x_{2}, x_{3}\right)$.

Applying $\varphi$, we get the following cycle corresponding to the triple logarithm $I_{1,1,1}\left(x_{1}, x_{2}, x_{3}\right)$ :

$Z_{x_{1}, x_{2}, x_{3}}=\left[1-\frac{1}{t}, 1-\frac{t}{x_{1}}, 1-\frac{t}{u}, 1-\frac{u}{x_{2}}, 1-\frac{u}{x_{3}}\right]+\left[1-\frac{1}{t}, 1-\frac{t}{u}, 1-\frac{u}{x_{1}}, 1-\frac{u}{x_{2}}, 1-\frac{t}{x_{3}}\right]$.

Here we have two parametrizing variables, $t$ and $u$, and $Z_{x_{1}, x_{2}, x_{3}} \in \mathcal{Z}^{3}(F, 5)$.

Example 5.3 In Figure 7, we give the sum of $F^{\times}$-deco trees associated to the weight 4 multiple logarithm $I_{1,1,1,1}\left(x_{1}, x_{2}, x_{3}, x_{4}\right)$.

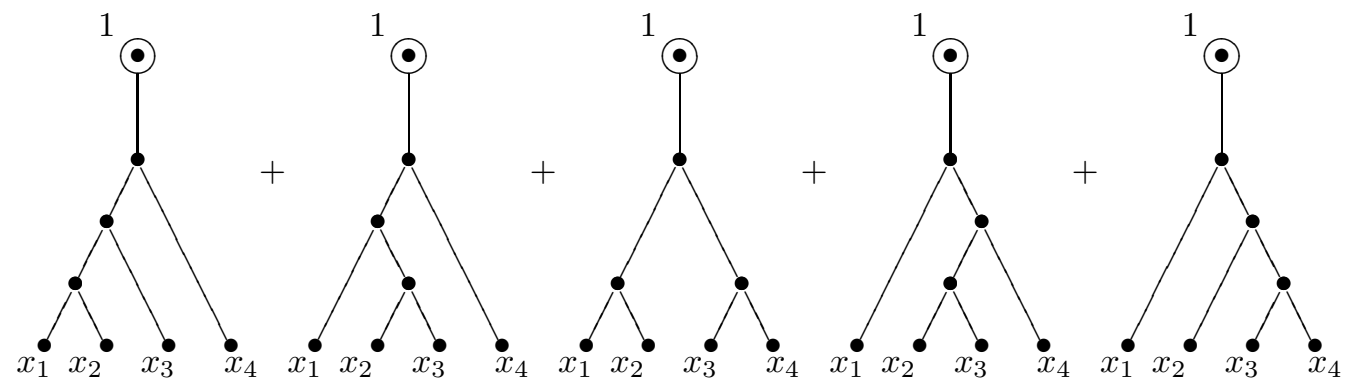

Figure 7: The sum of 5 trees corresponding to the multiple 4-logarithm.

One of the crucial properties of the elements $\tau\left(x_{1}, \ldots, x_{m}\right)$ is that the contributions of internal edges to the differential of $\tau\left(x_{1}, \ldots, x_{m}\right)$ cancel pairwise. This property ensures, cf. [3], that one can build from $\tau\left(x_{1}, \ldots, x_{m}\right)$ an element in the Hopf algebra $\chi_{\text {mot }}$ of [1]. Here is how the cancellations take place for $m=3$. The terms in $d \tau\left(x_{1}, x_{2}, x_{3}\right)$ coming from contracting the internal edges $e$ and $e^{\prime}$ cancel each other: $e$ is the third one in the (canonical counterclockwise) ordering of edges in the first tree, while $e^{\prime}$ is the second edge in the second tree, so the corresponding signs in the differential are opposite. 
This means that each term of $d \tau\left(x_{1}, x_{2}, x_{3}\right)$ is decomposable (in fact, since $\tau\left(x_{1}, x_{2}, x_{3}\right)$ consists of trivalent trees, each such term is a product of precisely two trees).

\section{Associating integrals to the multiple logarithm cycles}

So far we have not given a reason why we can consider the cycles associated to certain trees as "avatars" of multiple logarithms. In this section, we indicate how we can associate an integral to the cycles $Z_{x_{1}, x_{2}}$ and $Z_{x_{1}, x_{2}, x_{3}}$ which is nothing else than the integral presentation for the double and triple logarithm, respectively.

Following Bloch and Kříž, we embed the algebraic cycles into a larger setup of "hybrid" cycles which have both algebraic and topological coordinates as well as both types of differentials, and then apply the bar construction. We only consider "topological" variables $s_{i} \in[0,1] \subset \mathbb{R}$ subject to the condition $s_{i} \leqslant s_{j}$ if $i<j$, and taking the topological boundary $\delta$ for a cycle with topological dimension $r$ amounts to taking the formal alternating sum over the subvarieties where either $s_{k}=s_{k+1}$ for some $k=1, \ldots, r-1$ or $s_{1}=0$ or $s_{r}=1$.

Example 6.1 1. In order to bound $Z_{x_{1}, x_{2}}$, consider the algebraic-topological cycle parametrized by $t \in \mathbb{P}_{F}^{1}$ and $s_{1} \in \mathbb{R}, 0 \leqslant s_{1} \leqslant 1$, as

$$
\left[1-\frac{s_{1}}{t}, 1-\frac{t}{x_{1}}, 1-\frac{t}{x_{2}}\right]
$$

whose topological boundary terms are obtained by putting $s_{1}=0$ (which produces the empty cycle) or by $s_{1}=1$ which yields $Z_{x_{1}, x_{2}}$. Its algebraic boundary is given by

$$
\left[1-\frac{s_{1}}{x_{1}}, 1-\frac{s_{1}}{x_{2}}\right]-\left[1-\frac{s_{1}}{x_{1}}, 1-\frac{x_{1}}{x_{2}}\right]+\left[1-\frac{s_{1}}{x_{2}}, 1-\frac{x_{2}}{x_{1}}\right], \quad 0 \leqslant s_{1} \leqslant 1,
$$

where the last two terms are "negligible" for the following.

2. Consider the topological cycle parametrized by $0 \leqslant s_{1} \leqslant s_{2} \leqslant 1, s_{i} \in \mathbb{R}$, as

$$
\left[1-\frac{s_{1}}{x_{1}}, 1-\frac{s_{2}}{x_{2}}\right]
$$

whose boundary terms arise from setting $s_{1}=0, s_{1}=s_{2}$ or $s_{2}=1$, giving the empty cycle, $\left[1-\frac{s_{1}}{x_{1}}, 1-\frac{s_{1}}{x_{2}}\right]$ or $\left[1-\frac{s_{1}}{x_{1}}, 1-\frac{1}{x_{2}}\right]$, respectively.

What we are after is a cycle $\eta$ in this larger (algebraic-topological) cycle complex which bounds the cycle $Z_{x_{1}, x_{2}}$ i.e., such that $Z_{x_{1}, x_{2}}=(\partial+\delta) \eta$, since the "bounding process" will give rise to a purely topological cycle against which we can then integrate the standard volume form $(2 \pi i)^{-r} \frac{d z_{1}}{z_{1}} \wedge \cdots \wedge \frac{d z_{r}}{z_{r}}$ (here $r=2$ ), $z_{i}$ being a coordinate on the $i^{\text {th }}$ cube $\square_{\mathbb{R}}$. 
In fact, working modulo the "negligible" term $\left[1-\frac{s_{1}}{x_{1}}, 1-\frac{1}{x_{2}}\right]$ above we get that the full differential $D=\partial+\delta$ on the algebraic-topological cycle groups gives

$$
Z_{x_{1}, x_{2}}=D\left(\left[1-\frac{s_{1}}{t}, 1-\frac{t}{x_{1}}, 1-\frac{t}{x_{2}}\right]+\left[1-\frac{s_{1}}{x_{1}}, 1-\frac{s_{2}}{x_{2}}\right]\right)
$$

(two of the boundary terms cancel) and we associate to $Z_{x_{1}, x_{2}}$ the integral

$$
\begin{aligned}
\frac{1}{(2 \pi i)^{2}} \int_{\substack{\left[1-\frac{s_{1}}{x_{1}}, 1-\frac{s_{2}}{\left.x_{2}\right]} \\
0 \leqslant s_{1} \leqslant s_{2} \leqslant 1\right.}} \frac{d z_{1}}{z_{1}} \wedge \frac{d z_{2}}{z_{2}} & =\frac{1}{(2 \pi i)^{2}} \int_{0 \leqslant s_{1} \leqslant s_{2} \leqslant 1} \frac{d\left(1-\frac{s_{1}}{x_{1}}\right)}{1-\frac{s_{1}}{x_{1}}} \wedge \frac{d\left(1-\frac{s_{2}}{x_{2}}\right)}{1-\frac{s_{2}}{x_{2}}} \\
& =\frac{1}{(2 \pi i)^{2}} \int_{0 \leqslant s_{1} \leqslant s_{2} \leqslant 1} \frac{d s_{1}}{s_{1}-x_{1}} \wedge \frac{d s_{2}}{s_{2}-x_{2}} .
\end{aligned}
$$

Therefore we see that the cycle $Z_{x_{1}, x_{2}}$ corresponds - in a rather precise wayto the iterated integral $I_{1,1}\left(x_{1}, x_{2}\right)$.

Note that the three "negligible" terms encountered above can also be covered as part of a boundary if we introduce, following [1], yet another differential $\bar{d}$ (coming from the well-known bar construction), and in the ensuing tricomplex all the terms above are taken care of. With the usual bar notation | for a certain tensor product, the correct cycle is given by

$$
\begin{aligned}
{\left[1-\frac{s_{1}}{t}, 1-\right.} & \left.\frac{t}{x_{1}}, 1-\frac{t}{x_{2}}\right]+\left[1-\frac{s_{1}}{x_{1}}, 1-\frac{s_{2}}{x_{2}}\right] \\
& +\left(\left[1-\frac{s_{1}}{x_{1}} \mid 1-\frac{1}{x_{2}}\right]-\left[1-\frac{s_{1}}{x_{1}} \mid 1-\frac{x_{2}}{x_{1}}\right]+\left[1-\frac{s_{1}}{x_{2}} \mid 1-\frac{x_{1}}{x_{2}}\right]\right),
\end{aligned}
$$

and its image under the boundary $\partial+\delta+\bar{d}$ is precisely the "bar version" of $-Z_{x_{1}, x_{2}}$, given by $-Z_{x_{1}, x_{2}}+\left(\left[1-\frac{1}{x_{1}} \mid 1-\frac{1}{x_{2}}\right]-\left[1-\frac{1}{x_{1}} \mid 1-\frac{x_{1}}{x_{2}}\right]+\left[1-\frac{1}{x_{2}} \mid 1-\frac{x_{2}}{x_{1}}\right]\right)$. For details, we refer to [3].

\subsection{The integral associated to the triple logarithm}

In a similar fashion as for $Z_{x_{1}, x_{2}}$, we can find a bounding cycle $\gamma$ in the larger algebraic-topological groups. We picture the see-saw-like process with the main terms (all terms which do not appear in the diagram are "topologically decomposable" and therefore negligible for the final integral). In the cycles below, we have $t, u \in \mathbb{P}_{F}^{1},(t, u) \neq(0,0)$, and the range of the parameters $0 \leqslant s_{i} \leqslant 1$ is given via the inequalities $s_{i} \leqslant s_{j}$ for $i<j$. 


$$
\begin{aligned}
& {\left[1-\frac{1}{t}, 1-\frac{t}{x_{1}}, 1-\frac{t}{u}, 1-\frac{u}{x_{2}}, 1-\frac{u}{x_{3}}\right]} \\
& +\left[1-\frac{1}{t}, 1-\frac{t}{u}, 1-\frac{u}{x_{1}}, 1-\frac{u}{x_{2}}, 1-\frac{t}{x_{3}}\right]_{\nwarrow-\delta} \\
& {\left[1-\frac{s_{1}}{t}, 1-\frac{t}{x_{1}}, 1-\frac{t}{u}, 1-\frac{u}{x_{2}}, 1-\frac{u}{x_{3}}\right]} \\
& +\left[1-\frac{s_{1}}{t}, 1-\frac{t}{u}, 1-\frac{u}{x_{1}}, 1-\frac{u}{x_{2}}, 1-\frac{t}{x_{3}}\right] \\
& \swarrow \partial \\
& {\left[1-\frac{s_{1}}{x_{1}}, 1-\frac{s_{1}}{u}, 1-\frac{u}{x_{2}}, 1-\frac{u}{x_{3}}\right]} \\
& +\left[1-\frac{s_{1}}{u}, 1-\frac{u}{x_{1}}, 1-\frac{u}{x_{2}}, 1-\frac{s_{1}}{x_{3}}\right]_{\nwarrow^{-\delta}} \\
& {\left[1-\frac{s_{1}}{x_{1}}, 1-\frac{s_{3}}{u}, 1-\frac{u}{x_{2}}, 1-\frac{u}{x_{3}}\right]} \\
& +\left[1-\frac{s_{1}}{u}, 1-\frac{u}{x_{1}}, 1-\frac{u}{x_{2}}, 1-\frac{s_{3}}{x_{3}}\right] \\
& \swarrow \partial \\
& -\left[1-\frac{s_{1}}{x_{1}}, 1-\frac{s_{3}}{x_{2}}, 1-\frac{s_{3}}{x_{3}}\right] \\
& +\left[1-\frac{s_{1}}{x_{1}}, 1-\frac{s_{1}}{x_{2}}, 1-\frac{s_{3}}{x_{3}}\right] \\
& \nwarrow-\delta \\
& {\left[1-\frac{s_{1}}{x_{1}}, 1-\frac{s_{2}}{x_{2}}, 1-\frac{s_{3}}{x_{3}}\right]}
\end{aligned}
$$

Figure 8: A see-saw procedure to find the integral for the triple logarithm cycle.

The explanation of this diagram is as follows: the first two lines give the algebraic cycles for $Z\left(x_{1}, x_{2}, x_{3}\right)$; they are the images (modulo decomposable cycles) under $\delta$ of the algebraic-topological cycles shown in the next two lines (a real parameter $s_{1}$ enters). If we apply the full differential $D=\partial+\delta$ to the latter two cycles we obtain two new irreducible cycles, listed in the following two lines. Again we can bound the latter (under $\delta$ ) by two algebraic-topological cycles (a new parameter $s_{3}$ enters, and we have $0 \leqslant s_{1} \leqslant s_{3} \leqslant 1$; note that only the $\delta$-boundaries from putting $s_{1}=s_{3}$ are indecomposable). In the same fashion we consider the $\partial$-boundaries of the latter, winding up with $\gamma_{1,3}=$ $-\left[1-\frac{s_{1}}{x_{1}}, 1-\frac{s_{3}}{x_{2}}, 1-\frac{s_{3}}{x_{3}}\right]+\left[1-\frac{s_{1}}{x_{1}}, 1-\frac{s_{1}}{x_{2}}, 1-\frac{s_{3}}{x_{3}}\right], 0 \leqslant s_{1} \leqslant s_{3} \leqslant 1$ (note that the signs are opposite). In the final step, we see that the indecomposable part of the $\delta$-boundary of the purely topological cycle $\left[1-\frac{s_{1}}{x_{1}}, 1-\frac{s_{2}}{x_{2}}, 1-\frac{s_{3}}{x_{3}}\right]$ $\left(0 \leqslant s_{1} \leqslant s_{2} \leqslant s_{3} \leqslant 1\right)$ is precisely the above cycle $\gamma_{1,3}$. Therefore the sum over the five cycles on the right hand side of the picture bounds $-Z_{x_{1}, x_{2}, x_{3}}$, and the only cycle which gives a non-trivial integral against the standard volume form 
$(2 \pi i)^{-3} \frac{d z_{1}}{z_{1}} \wedge \frac{d z_{2}}{z_{2}} \wedge \frac{d z_{3}}{z_{3}}$ is the purely topological one, providing the integral

$$
-\frac{1}{(2 \pi i)^{3}} \int_{0 \leqslant s_{1} \leqslant s_{2} \leqslant s_{3} \leqslant 1} \frac{d s_{1}}{s_{1}-x_{1}} \wedge \frac{d s_{2}}{s_{2}-x_{2}} \wedge \frac{d s_{3}}{s_{3}-x_{3}},
$$

which is nothing else - up to the normalizing factor $(2 \pi i)^{-3}$ — than $I_{1,1,1}\left(x_{1}, x_{2}, x_{3}\right)$.

\section{Outlook}

We can also describe multiple polylogarithms $L i_{k_{1}, \ldots, k_{n}}\left(z_{1}, \ldots, z_{n}\right)$ in a similar fashion, but we need to introduce trees with two different kinds of external edges, and an accordingly modified forest cycling map gives us the associated algebraic cycles (cf. [3]).

A general construction in [1] based on the well-known bar construction applies to both DGA's, providing in particular a Hopf algebra structure on the $R$-deco forests, and $\varphi$ furthermore induces a morphism of Hopf algebras. Details for this, as well as the connection to the "motivic world", are given in [3].

\section{References}

1. Bloch, S., Kříž, I. Mixed Tate motives. Ann. of Math. (2) 140 (1994), no. 3, 557-605.

2. Bloch, S. Lectures on mixed motives. Algebraic geometry-Santa Cruz 1995, 329-359, Proc. Sympos. Pure Math., 62, Part 1, Amer. Math. Soc., Providence, RI, 1997.

3. Gangl, H.; Goncharov, A.B.; Levin, A. Multiple polylogarithms, polygons, trees and algebraic cycles. In preparation.

4. Gangl, H.; Müller-Stach, S. Polylogarithmic identities in cubical higher Chow groups. Algebraic K-theory (Seattle, WA, 1997), 25-40, Proc. Sympos. Pure Math., 67, Amer. Math. Soc., Providence, RI, 1999.

5. Goncharov, A.B. Polylogarithms in arithmetic and geometry. Proceedings of the International Congress of Mathematicians, Vol. 1, 2 (Zürich, 1994), 374-387, Birkhäuser, Basel, 1995.

6. Goncharov, A.B. The double logarithm and Manin's complex for modular curves. Math. Res. Lett. 4 (1997), no. 5, 617-636.

7. Goncharov, A.B. Galois groups, geometry of modular varieties and graphs. Talk at the Arbeitstagung Bonn, 1999. Available under http://www.mpimbonn.mpg.de/html/preprints/preprints.html

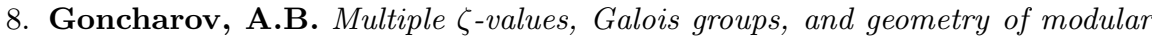
varieties. European Congress of Mathematics, Vol. I (Barcelona, 2000), 361-392, Progr. Math., 201, Birkhäuser, Basel, 2001. 